

\title{
Multiphoton Processes for Atoms in Intense Electromagnetic Fields
}

Lee A. Collins*, Joseph Abdallah, and George Csanak

\begin{abstract}
Lasers from table-top to giant ICF facilities that produce intense electromagnetic fields $\left(10^{14}-10^{21} \mathrm{~W} / \mathrm{cm}^{2}\right)$ have become important tools in probing the intricate nature of matter-radiation interactions. At such intensities, the laser field equals or exceeds that which binds electrons to an atom or molecule, and a new realm of physics opens in which perturbation theory may no longer suffice. We are developing several sophisticated techniques for treating atoms in such a regime, concentrating on two-photon X-ray absorption in intermediate-weight atoms and on laser-assisted electron-atom collisions. We perform most calculations in a time-independent frame in which field-free scattering formalisms can be invoked. We also investigate time-dependent methods in order to study transient effects. This is the final report of a three-year Laboratory-Directed Research and Development (LDRD) project at the Los Alamos National Laboratory (LANL).
\end{abstract}

\section{Background and Research Objectives}

With the advent of new laser technology capable of producing tightly-focused, shortduration pulses (ps to fs), electromagnetic (EM) fields can be generated with intensities of the order of $10^{14}$ to $10^{21} \mathrm{~W} / \mathrm{cm}^{2}$. At these intensities, the laser and atomic fields become comparable in strength, and we enter a new regime of atomic physics. Since the standard perturbative treatments for multiphoton processes may no longer be practical due to the large number of terms required, we must develop new techniques to probe this region. Phenomena such as "above-threshold" ionization (ATI), in which series of peaks separated by the photon energy are observed in the electron energy distribution, and large-scale multiple ionizations are unique features of this new realm. As intensities are increased, we expect that features such as relativistic effects may become important. In addition, as the pulse lengths become shorter (a few fs) and approach the order of a few cycles of the EM field, we anticipate that transient

*Principal investigator, e-mail: lac@lanl.gov 
effects will play a major role in the interpretation of the observations. For this case, standard steady-state models will eventually fail, and we shall have to develop fully time-dependent formulations to probe this regime. All of these processes may have additional applications to laser technologies and plasma modeling, in particular to the development of ultra fast X-ray sources by the use of short-pulse (subpicosecond) laser-produced plasmas.

Our objective in this LDRD project was to perform a theoretical study of the interaction of matter with intense electromagnetic radiation in which the energy in the field is comparable to that of the bound atomic electrons. Our principal endeavor centered on a basic understanding of multiphoton ionization of atoms by direct and indirect radiation from the new lasers, which produce very intense fields in confined areas with short pulses.

\section{Importance to LANL's Science and Technology Base and National R\&D Needs}

We collaborated closely with the experimental groups in Physics (P) and Chemical Science and Technology (CST) Divisions responsible for the short-pulse laser systems in order to supply theoretical support and guidance. The field is quite young, and therefore the full ramifications are still unclear. However, such intense matter-radiation interactions are known to be important in the design of short-wavelength lasers, in material damage, and in plasma modeling. Such mechanisms as coherent control of the interaction process can be accomplished through shaping the short-pulse. In addition, we have a collaboration with an experimental team consisting of members of CST and P Divisions and the University of New Mexico that plans to study multiphoton processes in $\mathrm{H}^{-}$at the Los Alamos Meson Physics Facility (LAMPF).

\section{Scientific Approach and Results to Date}

\section{a) Multiphoton Ionization of Atoms by X-ray sources}

In close support of the Los Alamos laser experiments, we have calculated[1] twophoton excitation and ionization cross sections from atomic species such as chlorine in the $\mathrm{X}$ ray regime. Since the $\mathrm{X}$-rays will be produced from a secondary source, the intensity range, although high, will still permit a perturbative formulation. We employ standard second-ordertime-dependent perturbation theory to determine the basic cross sections and transitions rates. The bound-bound and bound-free matrix elements are generated using the suite of Los Alamos atomic structure programs, CATS and GIPPER[2]. These programs allow the matrix elements to be treated at various levels of sophistication including Hartree-Fock and configuration 
interaction (CD). The sum over bound and continuum states is performed by the DalgarnoLewis technique that converts the summation to the solution of a second-order inhomogeneous differential equation. We have demonstrated that these sophisticated calculations predict ionization rates substantially larger than simple theories based on hydrogenic scaling. The higher rates bode well for the first experimental measurement of this multiphoton event. Such experiments are currently under way on the Trident Laser at Los Alamos. These detailed calculations will also greatly assist in the analysis of the experimental data as well as suggest other systems that would merit investigation. They will also greatly refine the region in which the search must be mounted for the Auger signature by which the two-photon process will be detected. The observation of multiphoton processes in the $\mathrm{X}$-ray region would be a first.

\section{b) Interaction of Atoms with Intense Fields}

The basic interaction of an atom with an intense electromagnetic field lies at the heart of any analysis of microscopic and macroscopic processes stimulated by the irradiation of matter, whether gas, solid or plasma, by high-intensity, short-pulse lasers. Therefore, gaining an intricate understanding of this basic process is essential of the modeling of more complex systems such as deposition and transport in high-energy, high-density plasmas. In addition, the intense field regime places extreme demands on theory since standard perturbative approaches no longer apply. To this end, we have solved the time-dependent Schroedinger equation[3], which describes the quantum mechanical interaction of an atom with an electromagnetic wave, converting to a time-independent prescription using Floquet theory in the Kramers-Henneberger(KH) gauge[4]. The resulting coupled equations bear a striking similarity to those for electron-molecule scattering, and we have applied the formidable techniques developed for molecular systems to this case[5]. The classical displacement, proportional to the electric field strength over the square of the frequency, serves an analogous role to the internuclear distance of a molecule. We have expanded the molecular linear algebraic method to encompass this wider domain of displacements[6].

We have employed the technique to calculate collisional parameters for electron-atom scattering in an intense field as well as multiphoton ionization rates for atoms and ions. The scattering calculations were the first using a linearly-polarized field for laser-assisted electron collisions with protons and revealed capture-escape resonances due to trapping in the bound states of $\mathrm{H}$. The field-free electron-proton collisional process exhibits no resonance structure. However, the inclusion of the laser field allows the emission of photons to drop the electron temporarily into a bound state of the compound $\mathrm{H}$ system. Absorbing photons from the field promotes the electron back into the continuum and gives rise to a distinct structure in the cross section. We have obtained angular distributions and partial cross sections for various 
multiphoton events for the scattered electrons from the rare gases [He, $\mathrm{Ne}]$ and compared results to recent experiments by Wallbank and coworkers[7]. Our results resolved several discrepancies between simpler theories and the experiments[8].

We have also calculated detachment rates for the negative ion of hydrogen $\mathrm{H}^{-}$ in regimes being explored by an experiment mounted at the Ground Test Accelerator (GTA) at LAMPF by a joint University of New Mexico[Bryant] and LANL[Strauss] group. The importance of this system stems from its simplicity, having only two electrons. Therefore, very sophisticated theoretical techniques can be brought to bear. In addition, the experimental results have a straightforward interpretation. We predicted that the two-photon process would yield observable signal, a point just verified by the experiments. This marks the first observation of multiphoton detachment in $\mathrm{H}^{-}$. In detachment in weak fields, only electrons with a energy equal to the difference of the photon and binding energies are observed. However, in the intense fields of the short-pulse lasers, a second set of electrons appear at an energy one-photon above the first set. This second peak in the continuum distribution due to the absorption of two photons marks an important new detachment mechanism. We accomplished this demonstration by extending our analysis procedures to encompass branching ratios or partial rates. We verified that the time-dependent and time-independent formulations gave results in very good agreement.

\section{References:}

[1] "Two-photon ionization of an inner shell of the $\mathrm{Cl}$ atom," J. Abdallah, L. Collins, G. Csanak, A. Petschek, and G. Schappert, Zeit. f Phys. (accepted, 1995).

[2] Theoretical Atomic Physics Code Development I-V, J. Abdallah, R.E.H. Clark, R.D. Cowan, G. Csanak, J.B. Mann, and S.P. Kramer, LA-11436-MI-IV and LA-11926 (1988-1990).

[3] "A simple model study of the interaction of an intense electric field with an atom," L.A. Collins, A.L. Merts, Phys. Rev. A 37, 245 (1988); ibid., Phys. Rev. A 40, 4127 (1989); "Multiphoton detachment of $\mathrm{H}^{-}$," L.A. Collins and A.L. Merts, Phys. Rev. A 45, 6615 (1992).

[4] "Multiphoton resonances in electron-proton scattering in an intense laser field," L.A. Collins and G. Csanak, Phys. Rev., 44, R5343 (1991); "Electron scattering by a potential in the presence of a strong radiation field," G. Csanak and L.A. Collins, Phys. Rev. A 47, 3240 (1993).

[5] "Linear Algebraic Methods," L.A. Collins and B.I. Schneider, Computational Methods for Electron Molecule Collisions (Plenum,1995). 
[6] "Electron scattering from the hydrogen molecular ion," L. Collins, B. Schneider,

D. Lynch, and C. Noble, Phys. Rev. A 52, 1310(1995).

[7] "Laser-assisted elastic electron-atom collisions," B. Wallbank and J.K. Holmes, J. Phys.

B 27, 1221(1994).

[8] "Electron scattering in intense fields," G. Csanak and L. Collins, ICPEAC

Proceedings(1995). 